\title{
Design of Reactance-to-Reactance Impedance Transformers Based on Conjugately Characteristic- Impedance Transmission Lines (CCITLs) and Meta-Smith Charts (MSCs)
}

\author{
Thanatcha SATITCHANTRAKUL ${ }^{1}$, Danai TORRUNGRUENG ${ }^{2}$ \\ ${ }^{1}$ Dept. of Electronics Engineering Technology, College of Industrial Technology, King Mongkut's University of \\ Technology North Bangkok, Bangkok, 10800, Thailand \\ ${ }^{2}$ Research Center of Innovation Digital and Electromagnetic Technology, Dept. of Teacher Training in Electrical \\ Engineering, Faculty of Technical Education, King Mongkut's University of Technology North Bangkok, Bangkok, \\ 10800, Thailand
}

Thanatcha.s@cit.kmutnb.ac.th,dtg@ieee.org

Submitted September 13, 2020 / Accepted April 22, 2021

\begin{abstract}
This paper proposes a novel technique to miniaturize the size of any reactance-to-reactance transformers (RRTs). These transformers are designed based on conjugately characteristic-impedance transmission lines (CCITLs) and Meta-Smith charts (MSCs). Note that the proposed technique can be effectively applied to popular microwave circuits; i.e., open-circuited and short-circuited tuning stubs as special cases. Numerical results are calculated, analyzed and compared with those of conventional stubs. In addition, the RRT prototype based on CCITLs is designed, simulated and measured to verify the proposed technique. It is found that the properly designed RRT prototype based on CCITLs can provide shorter electrical and physical lengths than those of the conventional RRT prototype indeed.
\end{abstract}

\section{Keywords}

Conjugately characteristic-impedance transmission line, reactance-to-reactance transformer, opencircuited stub, short-circuited stub, Meta-Smith charts

\section{Introduction}

One of the commonly used components in radio frequency and microwave systems is an impedance transformer [1]. The simplest transformer, called the quarter wave transformer, allows the power transfer between real different input and output impedances. However, these impedances are not always real in general. For example, a reactance can be occurred from reactive components especially at the load. Most of studies about reactance matching transformers are in the form of tuning stubs, which are widely used for size reduction of microwave devices [2-9]. For example, in [2-4] open-circuited stubs are proposed to miniaturize filters and couplers. Changing stub shapes (such as triangle and arc) is another way for size reduction [5-8]. In addition, stubs can also be shorten by using fractal structures [9]. Note that designing stubs can be classified as a reactance-to-reactance problem.

This paper proposes the novel method to reduce size of reactance-to-reactance transformers (RRTs) by using conjugately characteristic-impedance transmission lines (CCITLs). Note that CCITLs have been first proposed in 2004 [10] and widely applied later [11-17]. CCITLs are lossless transmission lines (TLs) with conjugate characteristic impedances for forward $\left(Z_{\mathrm{c}}^{+}\right)$and backward $\left(Z_{\mathrm{c}}^{-}\right)$ propagation directions. Interestingly, CCITLs can be applied to miniaturize microwave components [14-16]. To intuitively and effectively design CCITLs, Meta-Smith charts (MSCs) can be applied [17]. Like the Smith chart, MSCs usually provide a useful way with more physical insight of visualizing CCITL phenomenon and solving related problems effectively. It should be pointed out that the Smith chart is a special case of MSCs [17]. In this paper, the RRTs based on CCITLs will be designed using MSCs. It will be shown that the proposed RRT can provide shorter electrical length compared to that of the conventional RRT.

This paper is organized as follows. In Sec. 2, the theory of RRTs is presented. Section 3 shows results of some case studies with discussions along with simulation results of the RRT prototype using the new proposed technique. Finally, conclusions are presented in Sec. 4.

\section{Theoretical Background}

In this section, the theory of RRTs is derived for both conventional TLs and CCITLs based on the Smith chart and MSCs, respectively. 


\subsection{RRTs Implemented Using Standard TLs}

Figure 1 shows an RRT implemented using a standard TL to match a reactive load $\left(Z_{\mathrm{L}}=\mathrm{j} X_{\mathrm{L}}\right)$ to the input reactance $\left(Z_{\text {in }}=\mathrm{j} X_{\text {in }}\right)$. Note that $Z_{0,}, \beta$ and $\theta$ in Fig. 1 are the characteristic impedance, propagation constant and electrical length of the standard TL, respectively. In Fig. 1, the load $\left(\Gamma_{\mathrm{L}}\right)$ and input $\left(\Gamma_{\text {in }}\right)$ reflection coefficients for the standard TL are given as

$$
\begin{gathered}
\Gamma_{\mathrm{L}}=\frac{\mathrm{j} X_{\mathrm{L}}-Z_{0}}{\mathrm{j} X_{\mathrm{L}}+Z_{0}}, \\
\Gamma_{\text {in }}=\frac{\mathrm{j} X_{\text {in }}-Z_{0}}{\mathrm{j} X_{\text {in }}+Z_{0}} .
\end{gathered}
$$

This impedance matching problem can be solved intuitively using the Smith chart. Figure 2 illustrates the Smith chart calculation of an RRT using a standard TL for the cases of $X_{\mathrm{L}}<X_{\text {in }}$ and $X_{\mathrm{L}}>X_{\text {in }}$. Note that $x_{\mathrm{L}}$ and $x_{\text {in }}$ in Fig. 2 are the normalized $X_{\mathrm{L}}$ and $X_{\text {in }}$ with respect to $Z_{0}$, respectively. It should be pointed out that $\left|\Gamma_{\mathrm{L}}\right|=\left|\Gamma_{\text {in }}\right|=1$ (see (1) and (2)) implying that $\Gamma_{\mathrm{L}}$ and $\Gamma_{\text {in }}$ are located on the unit circle in the Smith chart. Figure 2(a) illustrates the Smith chart and associated electrical length $\theta$ in the case of $X_{\mathrm{L}}<X_{\text {in }}$, while the case of $X_{\mathrm{L}}>X_{\text {in }}$ is shown in Fig. 2(b).

In Fig. 2(a) $\left(X_{\mathrm{L}}<X_{\text {in }}\right), \Gamma_{\mathrm{L}}$ and $\Gamma_{\text {in }}$ are related to the following phase relationship (using (1) and (2)):

$$
\frac{\mathrm{j} X_{\mathrm{L}}-Z_{0}}{\mathrm{j} X_{\mathrm{L}}+Z_{0}}=\mathrm{e}^{\mathrm{j} 2 \theta} \frac{\mathrm{j} X_{\text {in }}-Z_{0}}{\mathrm{j} X_{\text {in }}+Z_{0}} .
$$

In addition, $\Gamma_{\mathrm{L}}$ and $\Gamma_{\text {in }}$ in Fig. $2(\mathrm{~b})\left(X_{\mathrm{L}}>X_{\text {in }}\right)$ can be expressed as

$$
\frac{\mathrm{j} X_{\text {in }}-Z_{0}}{\mathrm{j} X_{\text {in }}+Z_{0}}=\mathrm{e}^{\mathrm{j} 2(\pi-\theta)} \frac{\mathrm{j} X_{\mathrm{L}}-Z_{0}}{\mathrm{j} X_{\mathrm{L}}+Z_{0}} .
$$

It should be pointed out that (3) and (4) are identical mathematically. Rearranging (3) obtains the following equation:

$$
Z_{0}^{2} \tan \theta+\left(X_{\mathrm{L}}-X_{\text {in }}\right) Z_{0}+X_{\mathrm{L}} X_{\text {in }} \tan \theta=0
$$

where $0^{\circ} \leq \theta \leq 180^{\circ}$. For a given $\theta, Z_{0}$ of the standard TL can be readily found using (5) as

$$
Z_{0}=\frac{\left(X_{\text {in }}-X_{\mathrm{L}}\right) \pm \sqrt{\left(X_{\text {in }}-X_{\mathrm{L}}\right)^{2}-4 X_{\text {in }} X_{\mathrm{L}} \tan ^{2} \theta}}{2 \tan \theta} .
$$

Alternatively, for a given $Z_{0}, \theta$ of the standard TL can be obtained from (5) as

$$
\theta=\tan ^{-1}\left(\frac{\left(X_{\text {in }}-X_{\mathrm{L}}\right) Z_{0}}{Z_{0}^{2}+X_{\text {in }} X_{\mathrm{L}}}\right)
$$

Thus, an RRT implemented using a standard TL can be designed using either (6) or (7).

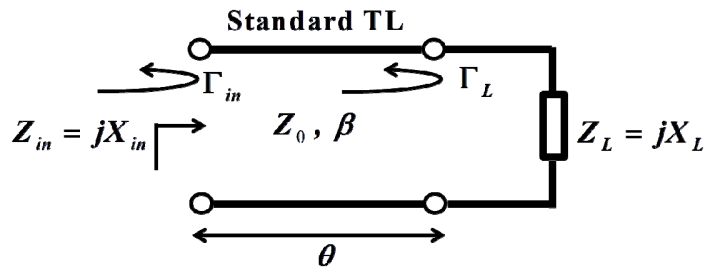

Fig. 1. An RRT implemented using a standard TL.

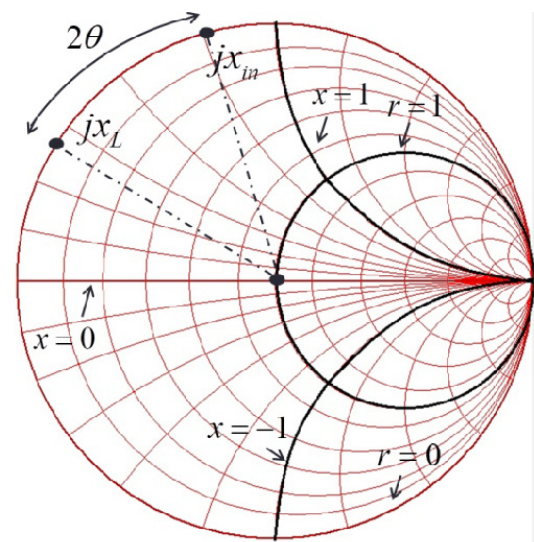

(a)

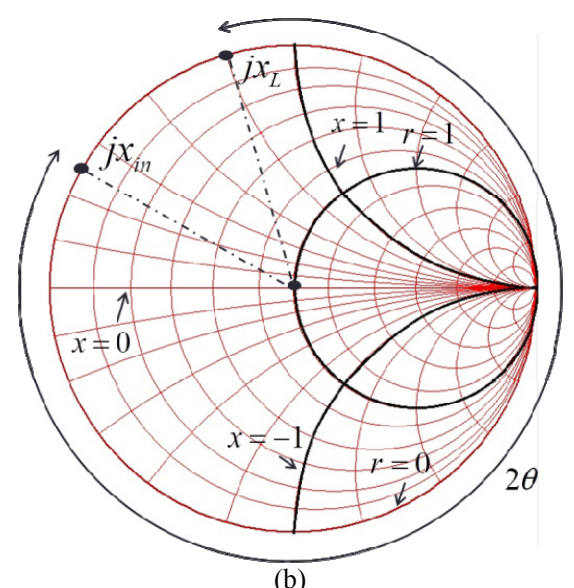

Fig. 2. The Smith chart calculation of an RRT using a standard TL: (a) $X_{\mathrm{L}}<X_{\text {in }}$, (b) $X_{\mathrm{L}}>X_{\text {in }}$.

\subsection{RRTs Implemented Using CCITLs}

Figure 3 presents an RRT implemented using a CCITL to match a reactive load $\left(Z_{\mathrm{L}}=\mathrm{j} X_{\mathrm{L}}\right)$ to the input reactance $\left(Z_{\text {in }}=\mathrm{j} X_{\text {in }}\right)$. Note that $Z_{\mathrm{c}}^{ \pm}, \beta$ and $\theta_{\mathrm{c}}$, in Fig. 3 are the characteristic impedances, propagation constant and electrical length of the CCITL, respectively. For convenience, $Z_{\mathrm{c}}^{ \pm}$of the CCITL are defined as [17]

$$
Z_{\mathrm{c}}^{ \pm}=\left|Z_{\mathrm{c}}^{ \pm}\right| \mathrm{e}^{\mp \mathrm{j} \phi}
$$

where $\left|Z_{\mathrm{c}}\right|$ and $\phi$ are the absolute value and the argument of $Z_{\mathrm{c}}^{-}$respectively, and $\phi$ is in the range of $-90^{\circ} \leq \phi \leq 90^{\circ}$ in this study. In Fig. 3 , the load $\left(\Gamma_{\mathrm{L}}\right)$ and input $\left(\Gamma_{\text {in }}\right)$ reflection coefficients for the CCITL can be expressed as [17] 


$$
\begin{gathered}
\Gamma_{\mathrm{L}}=\frac{\mathrm{j} X_{\mathrm{L}} Z_{\mathrm{c}}^{-}-\left|Z_{\mathrm{c}}^{ \pm}\right|^{2}}{\mathrm{j} X_{\mathrm{L}} Z_{\mathrm{c}}^{+}+\left|Z_{\mathrm{c}}^{ \pm}\right|^{2}}, \\
\Gamma_{\text {in }}=\frac{\mathrm{j} X_{\mathrm{in}} Z_{\mathrm{c}}^{-}-\left|Z_{\mathrm{c}}^{ \pm}\right|^{2}}{\mathrm{j} X_{\text {in }} Z_{\mathrm{c}}^{+}+\left|Z_{\mathrm{c}}^{ \pm}\right|^{2}} .
\end{gathered}
$$

This reactance-to-reactance matching problem can be solved graphically using the MSCs. There are four cases to consider for the design of RRTs implemented using CCITLs as shown in Figs. 4 to 7, where $x_{\mathrm{L}}$ and $x_{\text {in }}$ are the normalized $X_{\mathrm{L}}$ and $X_{\text {in }}$ with respect to $\left|Z_{\mathrm{c}}\right\rangle$, respectively. Note that $\left|\Gamma_{\mathrm{L}}\right|=\left|\Gamma_{\text {in }}\right|=1$ (see (9) and (10)) implying that $\Gamma_{\mathrm{L}}$ and $\Gamma_{\text {in }}$ are located on the unit circle in the MSCs. Each case and its MSC representation are shown below:

Case 1: $X_{\mathrm{L}}<X_{\text {in }}$ and $0^{\circ} \leq \phi \leq 90^{\circ}$ (see Fig. 4)

Case 2: $X_{\mathrm{L}}<X_{\text {in }}$ and $-90^{\circ} \leq \phi \leq 0^{\circ}$ (see Fig. 5)

Case 3: $X_{\mathrm{L}}>X_{\text {in }}$ and $0^{\circ} \leq \phi \leq 90^{\circ}$ (see Fig. 6)

Case 4: $X_{\mathrm{L}}>X_{\text {in }}$ and $-90^{\circ} \leq \phi \leq 0^{\circ}$ (see Fig. 7)

For Case 1, $\Gamma_{\mathrm{L}}$ and $\Gamma_{\text {in }}$ in Fig. 4 are related to the phase relationship as (using (8) to (10))

$$
\frac{\mathrm{j} X_{\mathrm{L}} \mathrm{e}^{\mathrm{j} \phi}-\left|Z_{\mathrm{c}}^{ \pm}\right|}{\mathrm{j} X_{\mathrm{L}} \mathrm{e}^{-\mathrm{j} \phi}+\left|Z_{\mathrm{c}}^{ \pm}\right|}=\mathrm{e}^{\mathrm{j} 2 \theta_{\mathrm{c}}} \frac{\mathrm{j} X_{\text {in }} \mathrm{e}^{\mathrm{j} \phi}-\left|Z_{\mathrm{c}}^{ \pm}\right|}{\mathrm{j} X_{\mathrm{in}} \mathrm{e}^{-\mathrm{j} \phi}+\left|Z_{\mathrm{c}}^{ \pm}\right|} .
$$

Rearranging terms in (11) results in

$$
\begin{aligned}
& \left|Z_{\mathrm{c}}^{ \pm}\right|^{2} \sin \theta_{\mathrm{c}}+\left(X_{\mathrm{L}} \cos \left(\theta_{\mathrm{c}}-\phi\right)\right. \\
& \left.-X_{\text {in }} \cos \left(\theta_{\mathrm{c}}+\phi\right)\right)\left|Z_{\mathrm{c}}^{ \pm}\right|+X_{\mathrm{L}} X_{\text {in }} \sin \theta_{\mathrm{c}}=0
\end{aligned}
$$

where $0^{\circ} \leq \theta_{\mathrm{c}} \leq 180^{\circ}$. For a given $\theta_{\mathrm{c}}, \mid Z_{\mathrm{c}}{ }^{ \pm}$of the CCITL can be found using (12) as (13)

$$
\begin{aligned}
\left|Z_{\mathrm{c}}^{ \pm}\right| & =\frac{X_{\text {in }} \cos \left(\theta_{\mathrm{c}}+\phi\right)-X_{\mathrm{L}} \cos \left(\theta_{\mathrm{c}}-\phi\right)}{2 \sin \theta_{\mathrm{c}}} \\
& \frac{ \pm \sqrt{\left(X_{\text {in }} \cos \left(\theta_{\mathrm{c}}+\phi\right)-X_{\mathrm{L}} \cos \left(\theta_{\mathrm{c}}-\phi\right)\right)^{2}-4 X_{\text {in }} X_{\mathrm{L}} \sin ^{2} \theta_{\mathrm{c}}}}{2 \sin \theta_{\mathrm{c}}} .
\end{aligned}
$$

Alternatively, for a given $\mid Z_{\mathrm{c}} \sharp, \theta_{\mathrm{c}}$ of the CCITL can be readily obtained from (12) as

$$
\theta_{\mathrm{c}}=\tan ^{-1}\left(\frac{\left(X_{\mathrm{in}}-X_{\mathrm{L}}\right) \cos \phi}{\left(X_{\mathrm{in}}+X_{\mathrm{L}}\right) \sin \phi+\left(\frac{\left|Z_{\mathrm{c}}^{ \pm}\right|^{2}+X_{\text {in }} X_{\mathrm{L}}}{\left|Z_{\mathrm{c}}^{ \pm}\right|}\right)}\right) .
$$

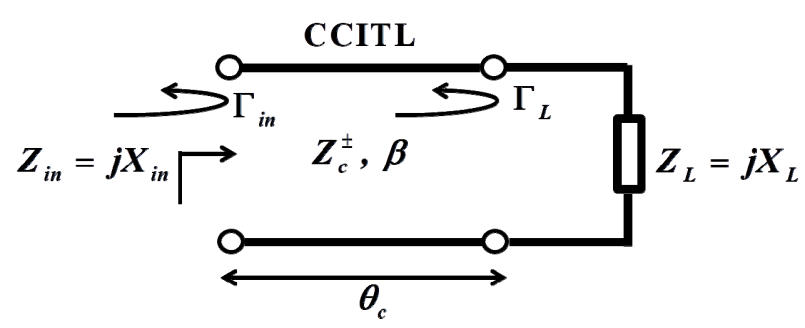

Fig. 3. An RRT implemented using a CCITL.

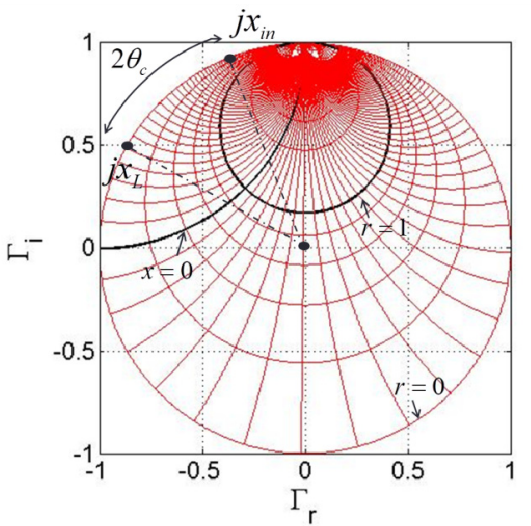

Fig. 4. The MSC $\left(\phi=45^{\circ}\right)$ for Case 1 with $0^{\circ} \leq \theta_{\mathrm{c}}<180^{\circ}$.

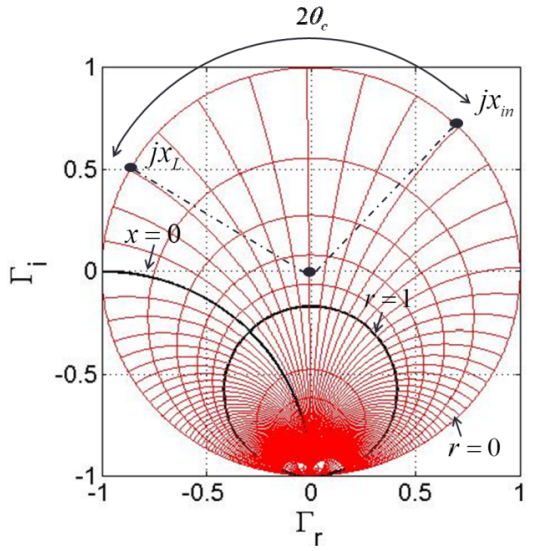

Fig. 5. The MSC $\left(\phi=-45^{\circ}\right)$ for Case 2 with $0^{\circ} \leq \theta_{\mathrm{c}}<180^{\circ}$.

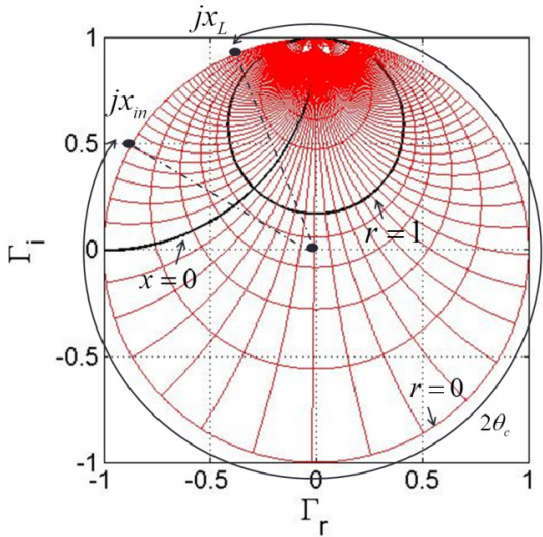

Fig. 6. The MSC $\left(\phi=45^{\circ}\right)$ for Case 3 with $0^{\circ} \leq \theta_{\mathrm{c}}<180^{\circ}$.

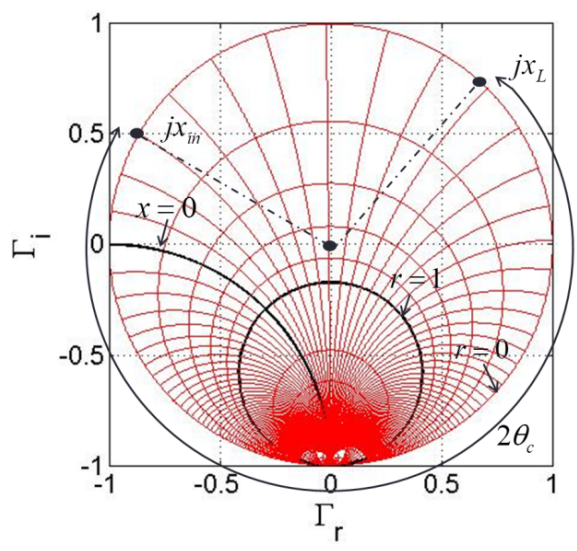

Fig. 7. The MSC $\left(\phi=-45^{\circ}\right)$ for Case 4 with $0^{\circ} \leq \theta_{\mathrm{c}}<180^{\circ}$. 
Thus, an RRT implemented using a CCITL can be designed using either (13) or (14). It should be pointed out that (13) and (14) are reduced to (6) and (7) when $\phi=0^{\circ}$, respectively. This comes from the fact that, when $\phi=0^{\circ}$, a CCITL becomes a standard TL and the MSCs become the Smith chart.

For Case 2, the relationship of $\Gamma_{\mathrm{L}}$ and $\Gamma_{\text {in }}$ in Fig. 5 are still the same as (11). Thus, an RRT implemented using a CCITL for this case can be designed using either (13) or (14) as well. In the cases of $X_{\mathrm{L}}>X_{\text {in }}$ (Cases 3 and 4), the starting equation is the same as (11) but different in the phase relationship as follows:

$$
\frac{\mathrm{j} X_{\text {in }} \mathrm{e}^{\mathrm{j} \phi}-\left|Z_{\mathrm{c}}^{ \pm}\right|}{\mathrm{j} X_{\text {in }} \mathrm{e}^{-\mathrm{j} \phi}+\left|Z_{\mathrm{c}}^{ \pm}\right|}=\mathrm{e}^{\mathrm{j} 2\left(\pi-\theta_{\mathrm{c}}\right)} \frac{\mathrm{j} X_{\mathrm{L}} \mathrm{e}^{\mathrm{j} \phi}-\left|Z_{\mathrm{c}}^{ \pm}\right|}{\mathrm{j} X_{\mathrm{L}} \mathrm{e}^{-\mathrm{j} \phi}+\left|Z_{\mathrm{c}}^{ \pm}\right|} .
$$

Note that (15) is identical to (11). Thus, an RRT implemented using a CCITL for Cases 3 and 4 can be designed using either (13) or (14) as well. Thus, all cases can be designed using the same design equations. Moreover, the RRTs using CCITLs will be implemented using microstrips to verify the proposed technique. In order to implement these, $Z_{\mathrm{c}}^{ \pm}$in (8) can be written in terms of the $A B C D$ parameters as [15-17]

$$
Z_{\mathrm{c}}^{ \pm}=\frac{\mp 2 B}{A-D \mp \mathrm{j} \sqrt{4-(A-D)^{2}}}
$$

where the $A B C D$ parameters in (16) are associated with the implemented RRT circuit using CCITLs. In addition, $\theta_{\mathrm{c}}$ in (14) can be written in terms of the $A B C D$ parameters as [15-17]

$$
\cos \theta_{\mathrm{c}}=\frac{A+D}{2} .
$$

Note that (16) and (17) are the main equations to design associated RRT circuit parameters to obtain the desired CCITL parameters $\left(Z_{\mathrm{c}}^{ \pm}\right.$and $\left.\theta_{\mathrm{c}}\right)$. For example, multisection TLs can be applied to implement CCITLs as discussed in details in [15]. In the next section, results of some case studies and the RRT prototype implemented using CCITLs are presented with discussions.

\section{Results and Discussions of Case Studies}

In Cases 1 and $2, X_{\mathrm{L}}$ and $X_{\text {in }}$ are assumed to be $50 \Omega$ and $100 \Omega$ respectively, while in Cases 3 and $4 X_{\mathrm{L}}$ and $X_{\text {in }}$ are set up to be $100 \Omega$ and $50 \Omega$, respectively. The unknown electrical length $\theta_{\mathrm{c}}$ is solved using (14) for given $\mid Z_{\mathrm{c}} t=50 \Omega, 150 \Omega$ and $250 \Omega$ by varying $\phi$ between $0^{\circ} \leq \phi \leq 90^{\circ}$ for Cases 1 and 3 and $-90^{\circ} \leq \phi \leq 0^{\circ}$ for Cases 2 and 4 . Figure 8 shows the plot of $\theta_{\mathrm{c}}$ versus $\phi$ with different $\left|Z_{\mathrm{c}}\right|$ for Case 1 . As $\phi$ increases, $\theta_{\mathrm{c}}$ tends to decrease monotonically for all values of $\left|Z_{\mathrm{c}}\right|$. In this cases, an RRT implemented using a CCITL can offer shorter electrical length compared to that of an RRT implemented using a standard TL $\left(\phi=0^{\circ}\right)$.

Figure 9 illustrates a similar plot of Fig. 8 but for Case 2. It is found that $\left|Z_{\mathrm{c}}^{\sharp}\right|=150 \Omega$ and $250 \Omega$ can offer shorter electrical length of RRTs for some values of $\phi$ only compared to that of an RRT implemented using a standard TL $\phi=0^{\circ}$. In addition Figure 10 shows a similar plot of Fig. 8 but for Case 3. As $\phi$ increases, $\theta_{\mathrm{c}}$ tends to increase monotonically for all values of $\mid Z_{\mathrm{c}} \pm$. In this case, an RRT implemented using a CCITL cannot offer shorter electrical length compared to that of an RRT implemented using a standard TL $\phi=0^{\circ}$. Finally, Figure 11 illustrates a similar plot of Fig. 8 but for Case 4. It is obvious that $\left|Z_{\mathrm{c}}^{\sharp}\right|=50 \Omega$ can offer shorter electrical length of RRTs, while $\mid Z_{\mathrm{c}}^{\sharp}=150 \Omega$ and $250 \Omega$ can offer shorter electrical length of RRTs for some values of $\phi$ only.

In addition, RRTs implemented using a standard TL and a CCITL are compared through the short-circuited $\left(X_{\mathrm{L}}=0\right)$ and open-circuited $\left(X_{\mathrm{L}} \rightarrow \infty\right)$ cases as well. For a fair comparison, let assume that $X_{\text {in }}$ is given and normalized with $Z_{0}=\mid Z_{\mathrm{c}}{ }^{\sharp}$. Following (7) for the standard TL and (14) for the CCITL, these equations can be reduced to (18) and (19) for the short-circuited case respectively, as shown below:

$$
\begin{gathered}
\tan \theta=\frac{X_{\text {in }}}{Z_{0}}=x_{\text {in }}, 0^{\circ} \leq \theta<180^{\circ}, \\
\tan \theta_{\mathrm{c}}=\frac{x_{\text {in }} \cos \phi}{x_{\text {in }} \sin \phi+1}, 0^{\circ} \leq \theta_{\mathrm{c}}<180^{\circ}
\end{gathered}
$$

where $x_{\text {in }}=X_{\text {in }} /\left|Z_{\mathrm{c}}^{ \pm}\right|$in (19), which is the same as $x_{\text {in }}$ in (18) since $Z_{0}=\left|Z_{\mathrm{c}}^{ \pm}\right|$in this case. Using (18) and (19), the following ratio can be computed for the short-circuited case:

$$
\frac{\tan \theta_{c}}{\tan \theta}=\frac{\cos \phi}{\tan \theta \sin \phi+1} \text {. }
$$

For the open-circuited case, (7) for the standard TL and (14) for the CCITL can be simplified to (21) and (22) respectively, as shown below:

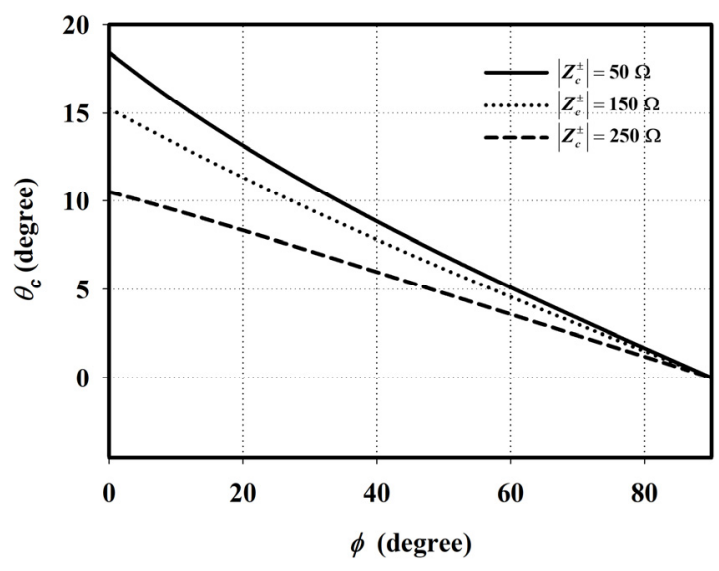

Fig. 8. Plot of $\theta_{\mathrm{c}}$ versus $\phi$ with different $\left|Z_{\mathrm{c}}{ }^{ \pm}\right|$for Case 1 . 


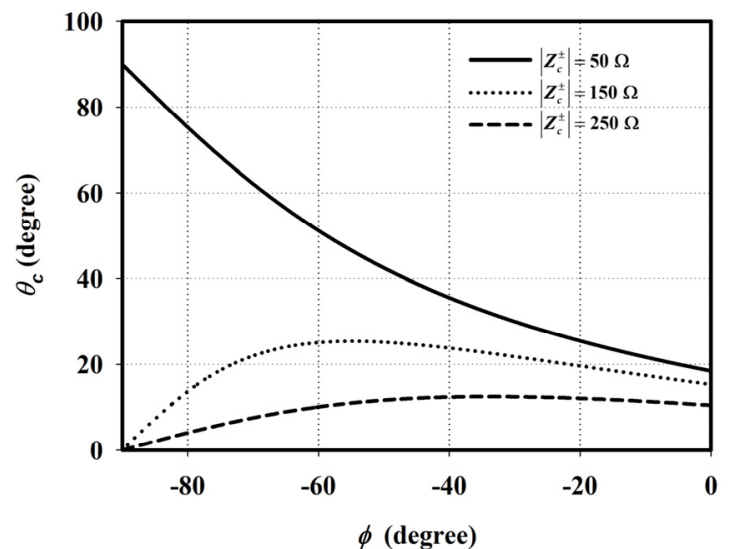

Fig. 9. Plot of $\theta_{\mathrm{c}}$ versus $\phi$ with different $\left|Z_{\mathrm{c}}^{ \pm}\right|$for Case 2 .

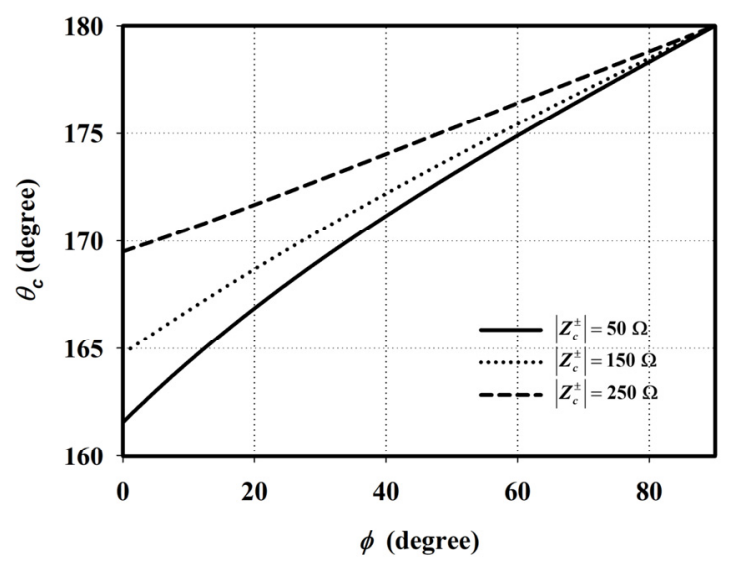

Fig. 10. Plot of $\theta_{\mathrm{c}}$ versus $\phi$ with different $\left|Z_{\mathrm{c}}^{ \pm}\right|$for Case 3 .

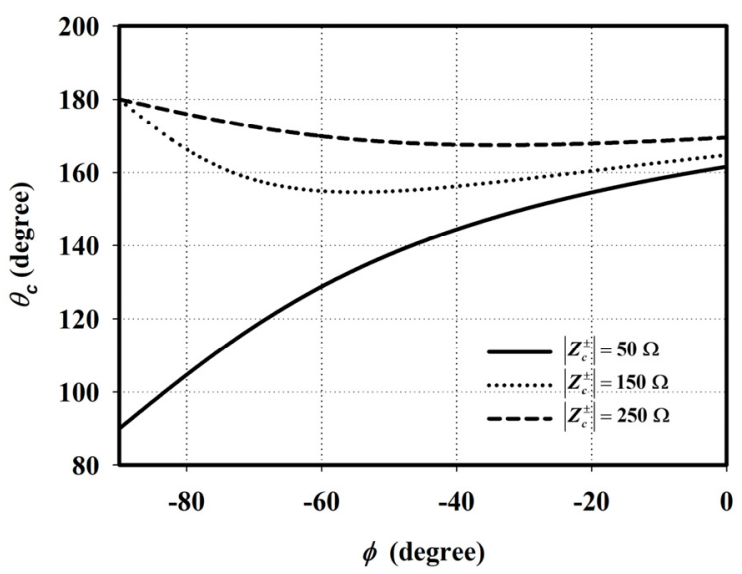

Fig. 11. Plot of $\theta_{\mathrm{c}}$ versus $\phi$ with different $\left|Z_{\mathrm{c}}^{ \pm}\right|$for Case 4 .

$$
\begin{aligned}
& \tan \theta=-\frac{Z_{0}}{X_{\text {in }}}=-\frac{1}{x_{\text {in }}}, 0^{\circ} \leq \theta<180^{\circ}, \\
& \tan \theta_{\mathrm{c}}=-\frac{\cos \phi}{x_{\text {in }}+\sin \phi}, 0^{\circ} \leq \theta_{\mathrm{c}}<180^{\circ} .
\end{aligned}
$$

Using (21) and (22), the following ratio can be calculated for the open-circuited case:

$$
\frac{\tan \theta_{\mathrm{c}}}{\tan \theta}=\frac{\cos \phi}{1-\tan \theta \sin \phi} .
$$

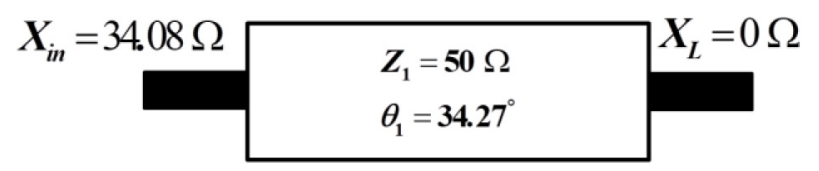

(a)

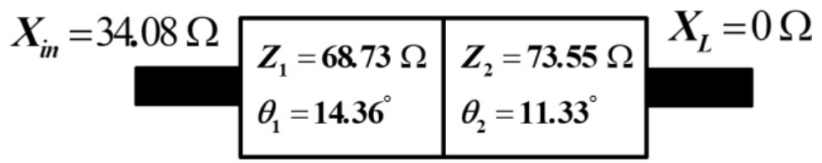

(b)

Fig. 12. Reactance matching circuit. (a) Standard TL, (b) Twosection TL.

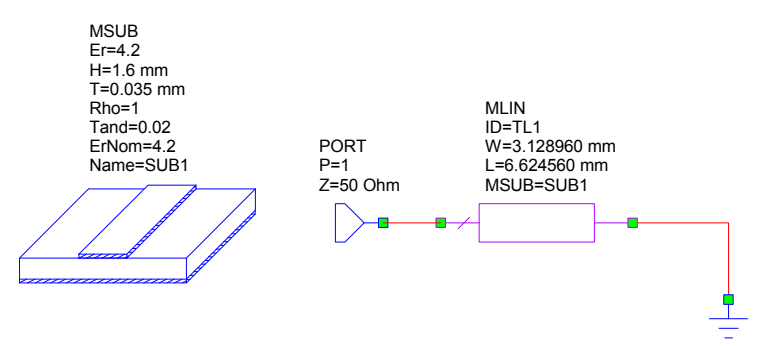

(a)
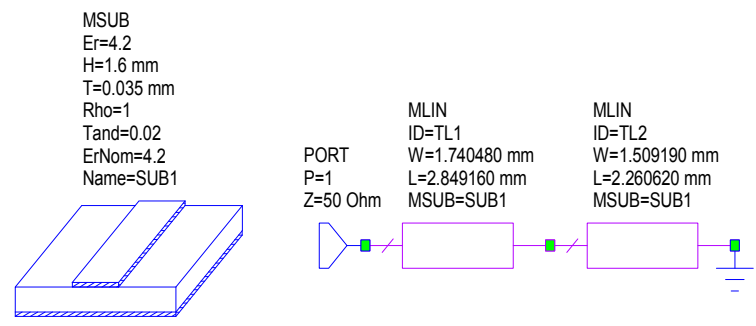

(b)

Fig. 13. Schematic design using microstrips. (a) Standard TL stub, (b) Two-section TL stub.

For illustration, given $X_{\text {in }}=34.08 \Omega$ and $X_{\mathrm{L}}=0 \Omega$, the short-circuited standard TL is designed using $Z_{0}=50 \Omega$ and $\theta=34.27^{\circ}$ as shown in Fig. 12 (a), simulated and compared with the short-circuited RRT stub based on the CCITL with $\left|Z_{\mathrm{c}}^{ \pm}\right|=Z_{0}$ and $\phi=25.2^{\circ}$. Note that both shortcircuited standard TL and RRT stub based on the CCITL are designed to operate at $2.4 \mathrm{GHz}$. Using (20), the ratio of the short-circuited case is approximately 0.70 , implied that the proposed RRT can reduce the stub electrical length indeed. Using a two-section TL to implement the CCITL, its TL parameters can be obtained, by optimizing (16) and (17) simultaneously for given $X_{\text {in }}=34.08 \Omega$ and $X_{\mathrm{L}}=0 \Omega$, as shown in Fig. 12 (b). For the first TL in Fig. 12 (b), its TL parameters are $Z_{1}=68.73 \Omega$ and $\theta_{1}=14.36^{\circ}$. For the second TL, its TL parameters are $Z_{2}=73.55 \Omega$ and $\theta_{2}=11.33^{\circ}$ as shown in Fig. 12(b).

The stubs in Fig. 12 can be realized using microstrips on the FR-4 substrate with the dielectric constant of 4.2 , the loss tangent of 0.02 , and the substrate thickness of $1.6 \mathrm{~mm}$. Using the AWR software [18], the schematic designs using microstrips are illustrated in Fig. 13(a) and (b) for the standard TL stub and the RRT implemented using CCITLs (two-section TL stub), respectively. Note that Figure 13 illustrates the width $(W)$ and length $(L)$ of 
each TL for both stub designs. For the standard TL stub, its dimension is $W=3.13 \mathrm{~mm}$ and $L=6.62 \mathrm{~mm}$. For the RRT implemented using CCITLs (two-section TL stub), its dimension is $W_{1}=1.74 \mathrm{~mm}, L_{1}=2.85 \mathrm{~mm}, W_{2}=1.51 \mathrm{~mm}$, and $L_{2}=2.26 \mathrm{~mm}$. Thus, the proposed RRT stub is clearly $22.81 \%$ shorter in physical length compared to that of the standard TL stub. In addition, Figure 14 shows the plots of the input reflection coefficient $\left(S_{11}\right)$ of the short-circuited stub on the Smith chart for both stub designs, where the frequency range is from $1 \mathrm{GHz}$ to $3 \mathrm{GHz}$. It is found that the associated input impedances of the standard short-circuited TL stub and the short-circuited RRT implemented using CCITLs (two-section short-circuited TL stub) are equal to $0.11+\mathrm{j} 34.27 \Omega$ and $0.14+\mathrm{j} 34.12 \Omega$, respectively. Note that their reactances are closed to the given $X_{\text {in }}=34.08 \Omega$, and their resistances are approximately zero as expected.

Next, the CST MWS software [19] is applied to simulate the coupling effects of stub and its connector. The input impedance results are $9.92+\mathrm{j} 327.34 \Omega$ and $3.60+\mathrm{j} 261.48 \Omega$ as shown in Fig. 15 (a) and (b) for the

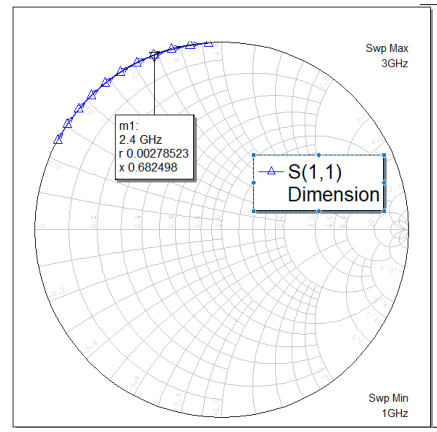

(a)

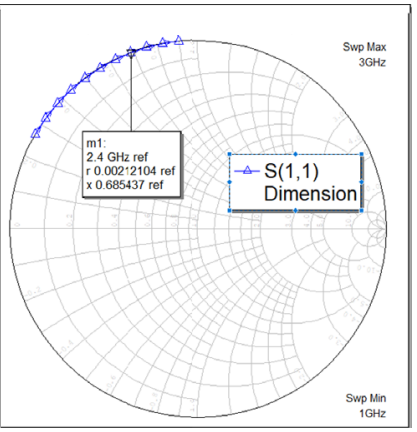

(b)
Fig. 14. Plot of the input reflection coefficient $S_{11}$ of the shortcircuited stub on the Smith chart from the AWR software. (a) Standard TL stub, (b) Two-section TL stub.
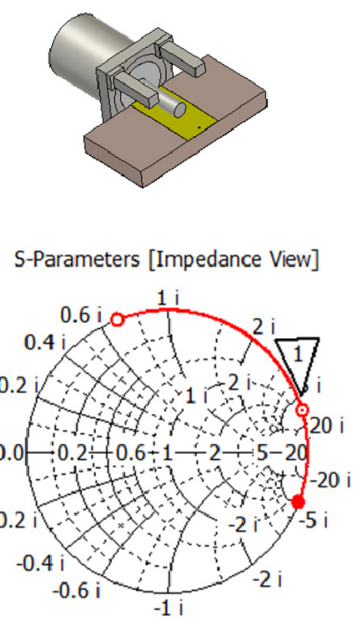

Q 2.400938 ( $9.920368,327.341323)$ Ohm

(a)

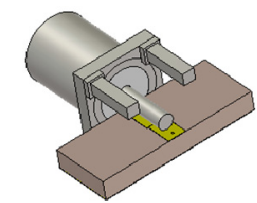

S-Parameters [Impedance View]

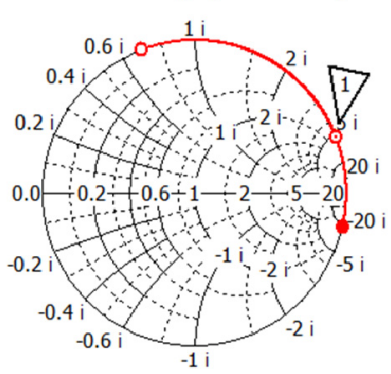

@ 2.400511 ( $3.605825,261.485682) \mathrm{Ohm}$

(b)
Fig. 15. Plot of the input reflection coefficient $\left(S_{11}\right)$ of the short-circuited stub on the Smith chart from the CST MWS software: (a) Standard TL stub, (b) Two-section TL stub with $25 \%$ electrical length reduction.
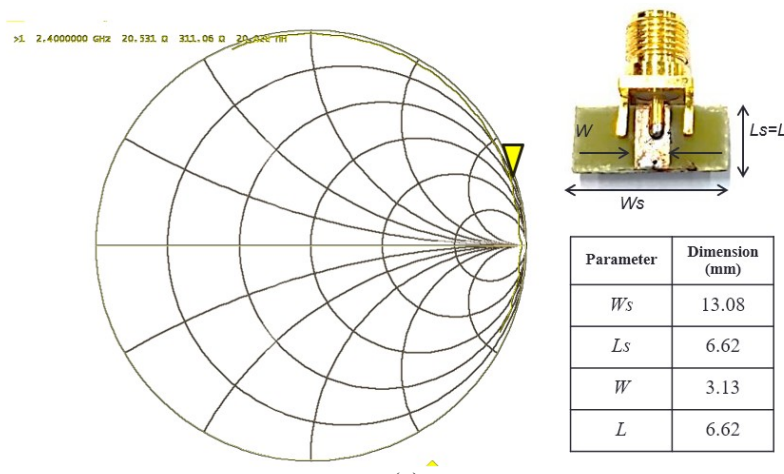

(a)
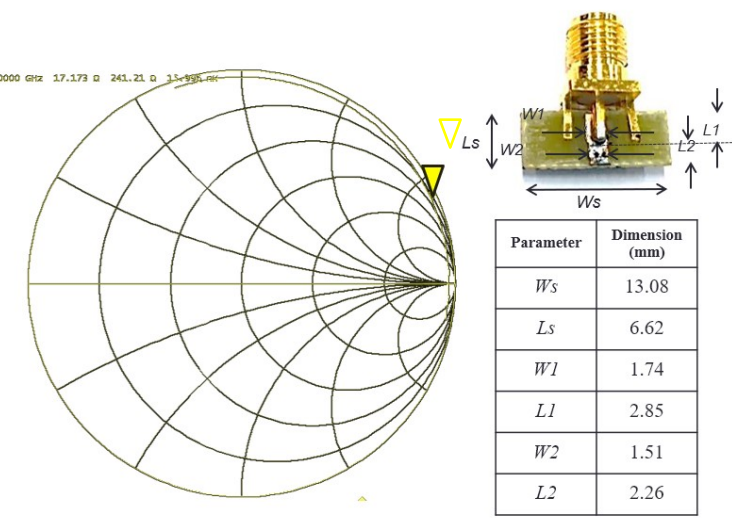

(b)

Fig. 16. Plot of the measured input reflection coefficient $\left(S_{11}\right)$ of the short-circuited stub on the Smith chart. (a) Standard TL stub, (b) Two-section TL stub with $25 \%$ electrical length reduction.

standard short-circuited stub and the short-circuited RRT using CCITL, respectively. Then, the prototype of the short-circuited RRT using CCITL is fabricated, and its input impedance is measured as $17.17+\mathrm{j} 241.21 \Omega$, compared with that of the standard short-circuited stub $(20.53+\mathrm{j} 311.06 \Omega)$ as displayed on the Smith chart in Fig. 16(b) and (a), respectively. It is seen that the simulation results from the CST MWS software are close to the measurement results indeed. The non-zero input resistances and shifting of either resistance or reactance of the AWR software, the CST MWS software and the measurement may be due to the coupling effects and the fact that the FR-4 substrate and the SMA connector are lossy. Thus, a properly designed RRT yields a desired input reactance, and can provide shorter electrical and physical lengths compared to those of the conventional RRT indeed.

\section{Conclusions}

In this paper, RRTs implemented using CCITLs are proposed to miniaturize the size of stubs and other RRTs. In addition, MSCs are applied as a useful tool to intuitively design and analyze RRTs implemented using CCITLs. For a practical example, the short-circuited RRT implemented using CCITLs is successfully designed, simulated and measured. It is found that properly designed RRTs, implemented using CCITLs, can provide shorter electrical and 
physical lengths compared to those of the conventional RRT indeed. In the future, the concept of this paper will be applied to other useful impedance transformation problems such as impedance-to-impedance transformers (IITs).

\section{Acknowledgments}

This research was funded by Thailand Science Research and Innovation Fund, and King Mongkut's University of Technology North Bangkok with Contract no. KMUTNB-BasicR-64-39.

\section{References}

[1] POZAR, D. M. Microwave Engineering. 4th ed. New Jersey: John Wiley \& Sons, 2012. ISBN: 0-471-64451-X

[2] KIM, T., CHOI, J. Miniaturized multi-section crossover with open stub. In IEEE International Symposium on Antennas and Propagation \& USNC/URSI National Radio Science Meeting. Vancouver (Canada), 2015, p. 1014-1015. DOI: 10.1109/APS.2015.7304910

[3] MAHARAJAN, R. K., KIM, N. Y. Miniature stubs-loaded square open-loop bandpass filter with asymmetrical feeders. Microwave and Optical Technology Letters, 2012, vol. 55, no. 2, p. 329-332. DOI: $10.1002 / \operatorname{mop} .27318$

[4] SAKAGAMI, I., HAGA, M., MUNEHIRO, T. Reduced branchline coupler using eight two-step stubs. IEE ProceedingsMicrowaves Antennas and Propagation, 1999, vol. 146, no. 6, p. 455-460. DOI: 10.1049/ip-map:19990785

[5] ZHANG, Y., SONG, H. Broadband miniaturized bandpass filter with circular stubs for compact wireless and mobile communication systems. Journal of Electromagnetic Waves and Applications, 2013, vol. 5, no. 3, p. 109-113. DOI: 10.4236/jemaa.2013.53018

[6] YU, J. J., CHEW, S. T., LEONG, M. S., et al. New class of microstrip miniaturised filter using triangular stub. Electronics Letters, 2001, vol. 37, no. 19, p. 1169-1170. DOI: 10.1049/el:20010793

[7] KUSUMA, Y., ISOZAKI, R. Compact and broadband microstrip band-stop filters with single rectangular stubs. Applied Sciences, 2019, vol. 9, no. 2, p. 1-12. DOI: 10.3390/app9020248

[8] XU, K., LI, M., LIU, Y., et al. Compact microstrip triple-mode bandpass filters using dual-stub-loaded spiral resonators. Radioengineering, 2017, vol. 26, no. 1, p. 23-29. DOI: $10.13164 /$ re.2017.0023

[9] MEZAAL, Y. S., ALI, J. K., EYYUBOGLU, H. T. Miniaturised microstrip bandpass filters based on Moore fractal geometry. International Journal of Electronics, 2014, vol. 102, no. 8, p. 1306-1319. DOI: 10.1080/00207217.2014.971351

[10] TORRUNGRUENG, D., THIMAPORN, C. Applications of the Z-YT-chart for nonreciprocal stub tuners. Microwave and Optical Technology Letters, 2005, vol. 45, no. 3, p. 259-262. DOI: 10.1002/mop.20789

[11] TORRUNGRUENG, D., THIMAPORN, C., LAMULTREE, S., et al. Theory of small reflections for conjugately characteristicimpedance transmission lines. IEEE Microwave and Wireless Components Letters, 2008, vol. 18, no. 10, p. 659-661. DOI: 10.1109/LMWC.2008.2003450
[12] TORRUNGRUENG, D., LAMULTREE, S., PHONGCHAROENPANICH, C., et al. In-depth analysis of reciprocal periodic structures of transmission lines. IET Microwave Antennas and Propagation, 2009, vol. 3, no. 4, p. 591-600. DOI: 10.1049/ietmap.2008.0205

[13] LIMSAENGRUCHI, S., SILAPUNT, R., TORRUNGRUENG, D. CCITL implementation using two-section microstrip transmission lines. In Proceedings of the 2012 IEEE International Symposium on Antennas and Propagation. Chicago (IL, USA), 2012, p. 1-2. DOI: 10.1109/APS.2012.6348901

[14] JONGSUEBCHOKE, I., AKKARAEKTHALIN, P., TORRUNGRUENG, D. Theory and design of quarter-wave-like transformers implemented using conjugately characteristicimpedance transmission lines. Microwave and Optical Technology Letters, 2016, vol. 58, no. 11, p. 2614-2619. DOI: 10.1002/mop.30120

[15] SATITCHANTRAKUL, T., CHUDPOOTI, N., AKKARAEKTHALIN, P., et al. An implementation of compact quarterwave-like transformers using multi-section transmission line. Radioengineering, 2018, vol. 27, no. 1, p. 101-109. DOI: $10.13164 /$ re.2018.0101

[16] SATITCHANTRAKUL, T., AKKARAEKTHALIN, P., SILAPUNT, R., et al. Compact wideband multi-section quarterwave-like transformers. Journal of Electromagnetic Waves and Applications, 2018, vol. 32, no. 15, p. 1911-1924. DOI: 10.1080/09205071.2018.1482239

[17] TORRUNGRUENG, D. Meta-Smith Charts and Their Potential Applications. La Vergne (TN): Morgan \& Claypool, 2010. DOI: 10.2200/S00276ED1V01Y201009ANT010 ISBN: 978-616-701909-6

[18] AWR Software. https://www.awr.com

[19] CST Microwave Studio. htttp://www.cst.com

\section{About the Authors}

Thanatcha SATITCHANTRAKUL was born in Bangkok, Thailand. She received her Ph.D. from King Mongkut's University of Technology Thonburi in 2018. Currently, she is working as a lecturer at King Mongkut's University of Technology North Bangkok, Bangkok, Thailand. Her research interests include antenna applications, transmission line applications and microwave sensors.

Danai TORRUNGRUENG (corresponding author) received his B.Eng. degree in Electrical Engineering (EE) from Chulalongkorn University, Bangkok, Thailand, in 1993. He obtained his M.S. and Ph.D. degrees in EE from The Ohio State University in 1996 and 2000, respectively. From 1995 to 2000, he was a Graduate Research Assistant (GRA) in the Department of Electrical Engineering, ElectroScience Laboratory of The Ohio State University. From 2002 to 2017, he worked in the Electrical and Electronic Engineering Department in the Faculty of Engineering and Technology at the Asian University, Chonburi, Thailand. At present, he is a Professor in the Department of Teacher Training in Electrical Engineering in the Faculty of Technical Education at King Mongkut's University of Technology North Bangkok, Bangkok, Thailand. In 2000, he won an award in the National URSI Student Paper competition at the 2000 National Radio Science Meeting in Boulder, 
Colorado. From 2004 to 2009, he invented generalized Smith charts, called T-charts or Meta-Smith charts, for solving several problems associated with conjugately characteristic-impedance transmission lines (CCITLs) and bicharacteristic-impedance transmission lines (BCITLs), including their useful applications in applied electromagnetics. He authored Meta-Smith Charts and Their Potential Applications (Morgan \& Claypool, 2010) and Advanced Transmission-Line Modeling in Electromagnetics (Charansanitwong Printing, 2012). His research interests are in the areas of electromagnetic sensors, fast computational electromagnetics, rough surface scattering, propagation modeling, electromagnetic wave theory, microwave theory and techniques and antennas. He is currently a senior member of the IEEE, and a member of the ECTI, where he had served as an ECTI technical chair in electromagnetics from 2014 to 2017. In addition, he served as a TPC co-chair of TJMW2016, a vice co-chair of TJMW2017 and the TPC chair of ISAP2017. Furthermore, he is a co-founder of the Innovative Electromagnetics Academy of Thailand (iEMAT) founded in 2013 (http://www.iemat.org/). 